\title{
Who Are the Internet Users, Mobile Internet Users, and Mobile-Mostly Internet Users?: Demographic Differences across Internet-Use Subgroups in the U.S.
}

\author{
Christopher Antoun \\ Institute for Social Research, Ann Arbor, USA, \\ antoun@umich.edu
}

\begin{abstract}
Survey researchers must now decide which data collection device or mix of devices is optimal for their Web survey (e.g., whether they will permit computers only or permit smartphones only). Their choice has implications for who can be observed and who cannot be observed. Yet there has been little research about the population subgroups that might be absent from different of types of Web surveys. This chapter takes a step in the direction of such research by exploring demographic differences across various subgroups of Internet users using data from a national telephone survey conducted in the US. Four overlapping groups are considered: the general population; those who go online using a computer; those who go online using a phone; and those who go online using mostly a phone, as opposed to other devices. In a novel approach to simplify the study of these groups, the process of using the Internet is modeled as a series of three transitions from one group to the next. This analysis sheds light on whether the effects of demographic characteristics are the same for each transition to a different level of Internet use. I also explore differences between these Internet use subgroups with respect to non-demographic survey variables after controlling for demographic differences.
\end{abstract}

How to cite this book chapter:

Antoun, C. 2015. Who Are the Internet Users, Mobile Internet Users, and Mobile-Mostly Internet Users?: Demographic Differences across Internet-Use Subgroups in the U.S.. In: Toninelli, D, Pinter, R \& de Pedraza, P (eds.) Mobile Research Methods: Opportunities and Challenges of Mobile Research Methodologies, Pp. 99-117. London: Ubiquity Press. DOI: http://dx.doi.org/10.5334/bar.g. License: CC-BY 4.0. 


\section{Keywords}

Web surveys, Internet access, mobile Web surveys, mobile Web access, mobilemostly users

\section{Introduction}

The rise of mobile devices is changing the way that people go online. People are increasingly using their mobile devices to complete online tasks that were once reserved for personal computers or laptops (e.g., checking email, reading the news, online shopping, or social networking). In addition, an increasing percentage of mobile users go online mostly using their mobile devices as opposed to their computers (Duggan \& Smith 2013).

For online survey researchers, this change presents both opportunities and challenges. The opportunities are mostly related to survey measurement. Smartphones provide ways to enhance measurement through the use of apps (e.g., survey apps, diary apps that prompt respondents) and other smartphone features (e.g., GPS, camera, Bluetooth-enabled sensors) (see AAPOR 2014). The challenges, on the other hand, have to do with reaching people in the general population and with the differences between those who are reached and those who are not reached. In the past, when personal computers were virtually the only way to access the Internet, Web coverage was only a function of the difference between those who went online using a computer and those who did not go online at all. However, the rapid rise of Internet-enabled personal devices has created other groups of Internet users, all of which have implications for coverage in online surveys. For example, if a traditional Web survey omits mobile phone users (by blocking them or using a non-optimized survey), then their absence from the sample might affect the accuracy of estimates based on Web surveys (Antoun \& Couper 2013). Mobile surveys might also affect coverage. If a mobile-only survey omits computer users (because it uses an app or takes advantage of the advanced features of smartphones), then their absence from the sample might bias estimates based on mobile-only surveys (Fuchs \& Busse 2009). The picture becomes more complicated when other devices, like tablets (not to mention e-readers, gaming consoles, etc.) are considered, and will no doubt become even more complex with the devices of the future (e.g., watches, wearable glasses, etc.).

Unfortunately, there has been little research about the characteristics of these different subgroups of Internet users, even though such knowledge might be informative for survey researchers who are grappling with which data collection device or mix of devices to use in order to reach the most people and who seek to understand which demographic groups may be underrepresented in their particular type of online survey (e.g., one that permit computers only, an app that permits smartphones only, or a hybrid or adaptive design approach 
that accommodates all devices). While some studies have estimated coverage error in surveys designed for computers (e.g., Mohorko, de Leeuw \& Hox 2013) and in surveys designed for mobile devices (e.g., Fuchs \& Busse 2009), this chapter takes a different approach. Using data from a Pew US national telephone survey, I explore demographic differences across various subgroups of Internet users. I distinguish between four overlapping groups: 1) the general population; 2) those who go online using a computer; 3) those who go online using a phone; and 4) those who go online mostly using a phone as opposed to other devices. Here I use the term 'go online' to refer not only to access but also to at least occasional use of a particular device to connect to the Internet. While there is also a small percentage of people who only use a phone but not a computer to go online, they make up such a small minority of US Internet users that they are not considered here (i.e., all phone users considered in this chapter also use a computer to go online). Tablets are not considered either, because the characteristics of their users do not have coverage implications for online surveys designed for mobile phones or computers; since tablets are not the focus of this chapter, from here on out I use the term 'mobile' to refer only to mobile phones.

As shown in Figure 1, when using the above classification the four groups are concentric in that each subsequent group is fully contained within the previous one(s). By definition, a member of group 4 (mobile-mostly user) is also a member of groups 3,2, and 1 (mobile user; computer user; general population); a member of group 3 (mobile user) is also a member of groups 2 and 1 (computer user; general population) but not necessarily group 4 (mobile-mostly user).

Because membership in a group is conditional on membership in the previous one(s), it is possible to conceptualize these as stages from which one can make up to three transitions, starting in group 1 and moving as far as group 4: that is, 1) an individual can transition from never going online to going online using a computer; 2) conditional on computer Internet use, they also have the opportunity to transition to going online using a phone; and 3) conditional to mobile Internet use, they can also transition to going online using mostly a phone. Each of these transitions represents movement from one side of a digital gap to the other side: one bridges a digital divide by starting to go online using a computer, a device divide by starting to go online using a phone, and what I call a usage divide (in the sense that they begin to use their phone more than other devices) by starting to go online using mostly a phone.

It should be noted that my focus is on Internet use in the US, where there has been a rapid shift in online use that matches these transitions. This framework would be less appropriate in other settings, such as developing countries, where a large proportion of people skipped the first transition altogether and started going online using a phone without ever having regularly used a computer (e.g., see Poushter, Bell \& Oates 2015). 


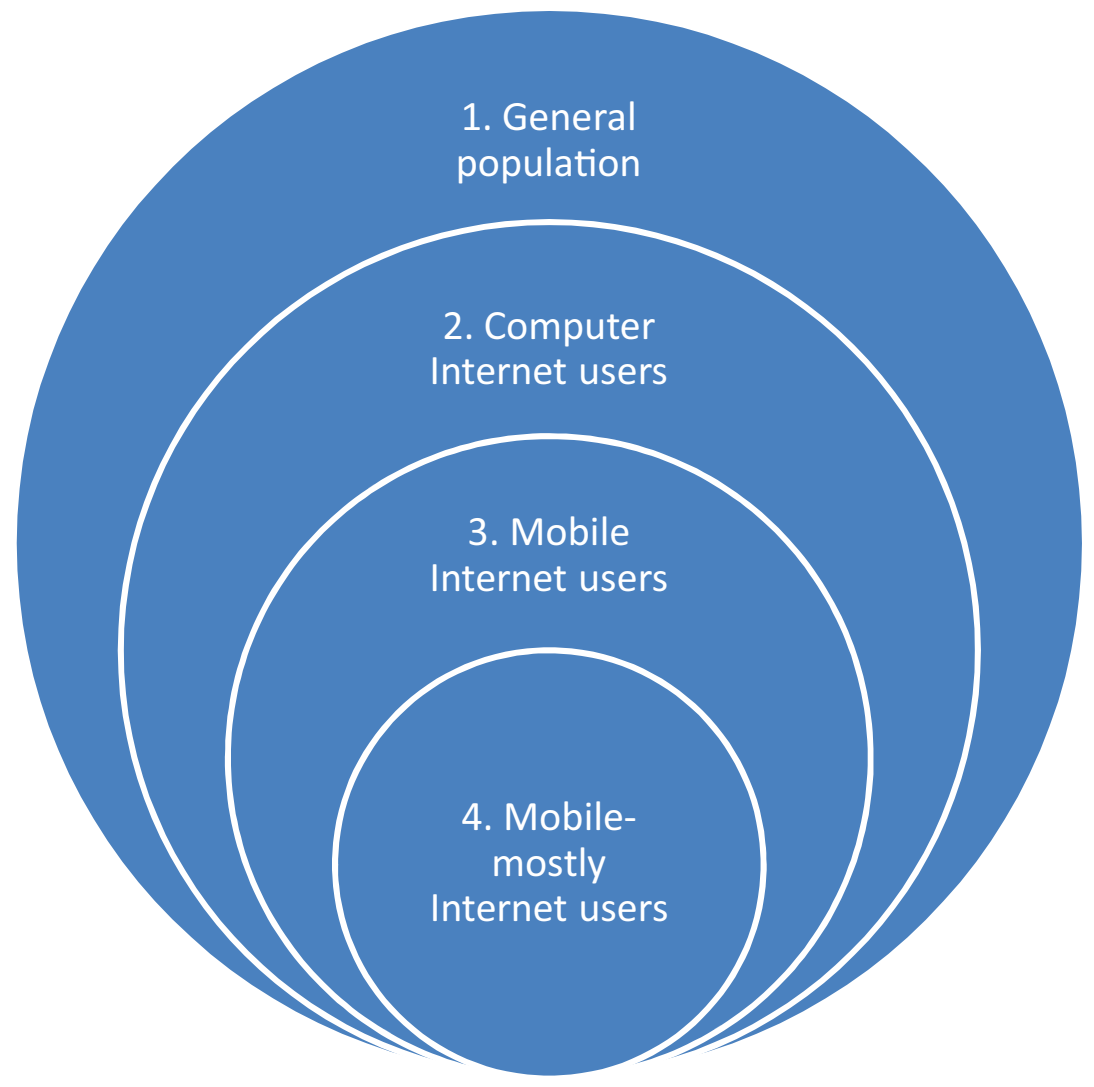

Figure 1: Internet Use Subgroups. Note: Figure not drawn to scale.

Conceptualizing Internet use in this way raises several questions about the effect of demographic characteristics at each transition. For example, age is known to play a role in Internet adoption, but does it also drive the transitions to going online using a phone and going online using mostly a phone? As for education, does its initial effect diminish with the transition to using a phone to go online or to using mostly a phone to go online? Race is also known to affect Internet adoption, but in what way, if any, is it also associated with the other two transitions? The primary objective of this chapter, therefore, is to investigate whether the effect of demographic characteristics are the same for each of these three transitions to a different level of Internet use. A secondary objective of this chapter is to investigate whether, conditional on the demographic variables, there is a relationship between substantive survey variables and these different levels of Internet use. The results will have implications for whether the omission of certain population subgroups in Web surveys might affect the accuracy of these surveys. 


\section{The Three Digital Divides}

As mentioned earlier, each of the three transitions under study in this chapter represents a kind of movement from one side of a digital gap to the other side. In this section, these three divides - the digital divide, device divide, and usage divide - are described in more detail.

\section{Digital divide}

Much attention has been paid to the distinction between those who go online and those who do not, i.e., the digital divide (Lenhart 2003). This was a concern as far back as 1995 (NTIA 1995), when a privileged group of only $3 \%$ of US residents had ever used the Internet (Pew 1995). Since then Internet use in the US has grown steadily and is now estimated to be as high as $85 \%$ (Zickuhr 2013). But there is evidence that a gap still exists between Internet users and nonusers. According to recent analyses of US survey data, Internet access decreases with age and increases with education, and non-Hispanic Black and Hispanic people are more likely to have access to the Internet than White people (Bethlehem 2010; Tourangeau, Conrad \& Couper 2013). For online survey practitioners, uneven Internet use across population groups has implications for coverage errors, because those on one side of the divide differ from those on the other side with regard to survey variables of interest such as health ratings and voting behavior (Bethlehem 2010; Couper et al. 2007; Dever, Rafferty \& Valliant 2008; Mohorko, de Leeuw \& Hox 2013).

\section{Device divide}

While the digital divide is still present, another type of divide has emerged, the so-called 'device divide' (Pearce \& Rice 2013). This divide makes a distinction between those who go online using their phones and those who go online but not using their phone. The number of mobile Internet users varies widely by country and region but appears to be growing in almost all places (for a more detailed overview, see Revilla et al. 2014). Just in the past few years, the percentage of mobile Internet users has recorded notable growth and is now estimated to be at $43 \%$ in Europe (Eurobarometer data retrieved from Metzler \& Fuchs 2014) and 55\% in the US (Duggan \& Smith 2013). But as with the digital divide, there is evidence that those on one side of the device divide differ from those on the other side. Using US national telephone surveys, Smith (2012) reports that mobile Internet users are younger, better educated, and more likely to be Black or Hispanic compared to those who do not use the mobile Internet. Using a face-to-face survey conducted in 33 European countries (Eurobarometer), Fuchs and Busse (2009) report similar differences: mobile Internet users are 
younger, more likely to be male, and more likely to be single. The extent of the differences for non-demographics variables is still unclear.

\section{Usage divide}

Finally, a usage divide between those who go online mostly using their phones and those who go online mostly using their computers is emerging among mobile Internet users. This transition has been highlighted in several recent reports produced by survey organizations (e.g., Duggan \& Smith 2013; Smith 2012; Townsend \& Rios 2011). It occurs when an individual begins to forgo using their computer in favor of their phone. For multi-device users, the choice of device for online activities might depend on several factors such as the complexity and length of the task (Robinson 2014), the time of day (Lipsman \& Aquino 2013), or whether they are at home or at work. With this in mind, it may be difficult for respondents to report their general go-to device for online browsing; nonetheless, when asked to do so they increasingly report choosing their phones. In just the past few years, the percentage of mobile-mostly users in the US has nearly doubled, jumping from 8\% in 2011 to 15\% in 2013 (Duggan \& Smith 2013). Users who prefer to use mobile phones for online tasks may be especially likely to take online surveys using their phone (de Bruijne \& Wijnant 2014), whether mobile use was intended by the designers of the survey or not (Peterson 2012). Of course, mobile-centric status does not mean that one cannot complete a survey using other devices - users can still make an exception and answer a survey on a computer. As with the other two divides, there is some evidence of differences between those on either side of the division. Duggan and Smith (2013) report that young adults, non-Whites, the less well educated, and the less affluent are more likely to go online using mostly their phones rather than other devices. This is perhaps a reflection of high-education individuals wanting to complete more complicated online tasks (e.g., banking, graphic design, and so forth) on a computer, and not on a phone (Robinson 2014). Little is known, however, about the differences between mobile-mostly Internet users and computer-mostly Internet users on non-demographic survey variables.

This chapter will explore demographic differences not only within each one of these three divides, but also across them. Given the evidence that technology use is generally higher among younger, highly educated, and more affluent persons, I expect to find several consistent effects across the three divides, including: an age effect - Internet use declines with age; an education effect - Internet use increases with education; and an income effect - Internet use increases with income. As mentioned earlier, I will also test for differences between these groups with regards to survey variables of interest (after controlling for demographic differences), because any significant differences could have coverage error implications for Web surveys. 


\section{Methods}

Data were analyzed from one year (2012) of the Pew Internet and American Life Project's Spring Tracking Study. This was a project of the Pew Research Center, a nonpartisan think tank based in Washington, DC. The study consisted of a US national telephone survey of adults aged 18 and older that used a dual-frame landline and cellular random digit dial (RDD) sample. Interviews were conducted in English and Spanish. The questionnaire contained approximately 60 questions, mostly on the topic of technology use. Data collection was carried out by Princeton Survey Research Associates International. There were 2,254 completed interviews (1,351 landline and 903 cell) and the response rate was $11 \%$. The low response rate can be partially attributed to the larger trend of declining response rates in US telephone surveys (Curtin, Presser \& Singer 2005). While higher response rates are desirable, this low response rate is not necessarily an indicator of nonresponse bias in the estimates (Groves \& Peytcheva 2008), especially since the topic of the survey (i.e., technology use) was not made explicit in the introduction that was read by interviewers. To know about Internet users and non-users, one needs data on both groups, and an interviewer-administered survey allows for this in a way that a Web survey does not. Of course, there is the chance of coverage error in the estimates based on telephone surveys because people without phones were excluded, but they are estimated to make up a relative small proportion (about 2\%) of the US population (Blumberg \& Luke 2013). To help reduce these potential biases and make inferences to the general US population of adults (aged 18 and older), all analyses presented here were weighted using the combined first-stage weights (to account for overlapping sample frames and different household sizes) and second-stage poststratification weights (see Duggan \& Rainie 2012 for information about the estimation of weights).

In my analysis, I used the following demographics variables: age (18-24 versus $25-34,35-44,45-54,55-64$, or 65 and older); education (less than high school graduate versus high school graduate, some college/Associate's degree, or college graduate or more); income (less than US $\$ 30,000$ versus US $\$ 30,000-$ US $\$ 75,000$, more than US $\$ 75,000$, or don't know/refused); gender (male versus female); and race/ethnicity (White/not Hispanic versus, Black/not Hispanic, Hispanic, or other). These variables were chosen because they are used for postratification weighting by Pew and because they are commonly used to describe differences between adults with and without Internet access. I also used the following three non-demographic survey variables: quality of life (excellent/ very good/good versus fair/poor/don't know/refused); social trust ('most people can be trusted' versus 'you can't be too careful'/it depends'/don't know/ refusal); tablet user ('yes' versus 'no'/don't know/refusal). These variables serve as examples of substantive variables that might be measured using a Web survey. I chose these three items because they are of substantive interest to Pew, and because they were administered to all respondents, regardless of whether 
or not they have Internet (some items in the survey were not administered to non-Internet users).

There are a number of different ways to measure how people go online, and consideration can be given to access versus use, individual use versus household use, location of use, and so forth. As mentioned earlier, I focus not on access only but on at least occasional use of a particular device to connect to the Internet. I defined Internet use at the individual level and not the household level. Also, I did not consider the places where people use the Internet, even though some people's online time may be concentrated at work or at home (or at the library or a coffee shop for that matter). In my analysis, an individual who goes online using a computer is someone who answered 'yes' to either of two questions - 'Do you use the internet, at least occasionally?' or 'Do you send or receive email, at least occasionally?' - and who reported owning either a 'desktop computer' or a 'laptop computer'. An individual who goes online using a phone is someone who answered 'yes' to either of these questions: 'Do you ever use your cell phone to send or receive email?' or 'Do you ever use your cell phone to access the internet?' As noted earlier, all phone users considered in this analysis also use a computer to go online (the small number of people ( $n=69$ or $3.1 \%$ of the sample) who only use a phone but not a computer to go online are excluded). The excluded mobile-only respondents did not differ reliably from the mobile-mostly respondents in terms of gender or race/ethnicity, but they were slightly older than the mobile-mostly respondents. Finally, an individual who goes online using mostly a phone is someone who answered 'mostly on a cell phone' rather than 'mostly on something else', 'both equally', 'depends', or 'don't know' to the following question: 'Overall, when you use the internet, do you do that mostly using your cell phone or mostly using some other device like a desktop, laptop or tablet computer?'

\section{Statistical analysis}

As mentioned earlier, I consider four overlapping groups of Internet users. I take advantage of the fact that membership in one group is conditional on membership in the previous one(s), and I model the process of gaining Internet access as a series of binary choices by fitting three sequential logistic regression models that are estimated by conditioning on the appropriate subsamples of the data. For model 1 , I used the full sample $(n=2254)$ to model computer Internet use vs. non-use. In this group, $74.1 \%$ of respondents report going online using a computer (75.2\% in the weighted analysis). For model 2, I condition on computer Internet use $(n=1671)$ and then model mobile Internet use vs. non-use (i.e., the conditional probability of going online using a phone). In this subsample, $61.0 \%$ of respondents report going online using a phone $(66.9 \%$ in the weighted analysis). For model 3, I condition on mobile Internet use ( $n=1019)$ and model mobile-mostly use vs. computer-mostly use (i.e., the conditional 
probability of going online using mostly a phone). In this subsample, $16.4 \%$ of respondents report going online using mostly a phone $(21.1 \%$ in the weighted analysis).

\section{Results}

For this analysis, I first fit the multivariate models using only demographic variables. The results will shed light on demographic differences between users and non-users at each level of Internet use. They will also reveal whether the effects of demographic characteristics are the same for each of the three transitions to a different level of Internet use. I then refit the models using both demographics variables and non-demographics variables to see if the additional variables are associated with being a particular type of Internet user over and above the demographic predictors.

\section{Demographics-only models}

The series of conditional logic models and their odds ratios are presented in Table 1. Model 1 reveals that all five demographic variables were significantly associated with computer Internet use. Such users are younger, more likely to be female, better educated, more likely to be White than Black or Hispanic, and more affluent than non-users. I examined the bivariate relationships of these variables to find that, for age, computer Internet use decreased in a monotonic way from $87.9 \%$ among those respondents aged $18-24$ to $50.6 \%$ among those aged 65 and older. Computer Internet use increased monotonically with education from $43.1 \%$ among those with less than a high school degree to $92.2 \%$ among college graduates.

Model 2 reveals that (conditional on computer Internet use), mobile Internet users are younger, better educated, and more likely to be Black or Hispanic than White, and more affluent than mobile Internet non-users. As with computer Internet use, mobile Internet use decreased with age from $77.9 \%$ among those respondents aged 18-24 to $19.4 \%$ among those aged 65 and older, but it increased with education from $42.5 \%$ among those with less than a high school degree to $67.2 \%$ among college graduates. Mobile Internet use among Black and Hispanic respondents $(64.2 \%$ and $63.5 \%$, respectively) was higher than among White respondents (52.7\%).

Model 3 reveals that (conditional on mobile Internet use), mobile-mostly Internet users are younger, and more likely to be Black than computermostly Internet users. As with the other types of Internet use, mobile-mostly use decreased with age from $26.6 \%$ among those respondents aged 18-24 to less than $2 \%$ for each of the two oldest age groups (55-64 and 65 and older). Mobile-mostly Internet use increased with education up to a point, from $8.2 \%$ 


\begin{tabular}{|c|c|c|c|c|c|c|c|c|c|c|c|c|c|c|c|}
\hline 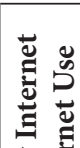 & 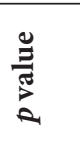 & $\begin{array}{l}\vec{\Xi} \\
\stackrel{8}{0} \\
\dot{0} \\
v\end{array}$ & & & & & & & $\begin{array}{l}2 \\
0 \\
0\end{array}$ & & & & & $\underset{\widetilde{\jmath}}{\tilde{0}}$ & \\
\hline 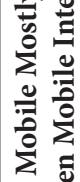 & $\begin{array}{l}\text { J } \\
\text { 우 } \\
\text { in }\end{array}$ & & 1 & $\begin{array}{c}\infty \\
n \\
\\
\tilde{n} \\
\tilde{n} \\
0\end{array}$ & $\begin{array}{l}\widehat{N} \\
\hat{0} \\
0 \\
0 \\
1 \\
0 \\
0\end{array}$ & $\begin{array}{l}\widehat{\tilde{I}} \\
\tilde{0} \\
\hat{0} \\
\dot{0} \\
\dot{0}\end{array}$ & $\begin{array}{l}0 \\
0 \\
0 \\
0 \\
\hat{0} \\
0 \\
0\end{array}$ & 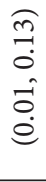 & & 1 & $\begin{array}{l}\hat{n} \\
\hat{n} \\
\hat{2} \\
\hat{0} \\
0\end{array}$ & 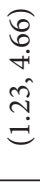 & 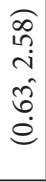 & & 1 \\
\hline 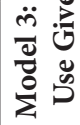 & 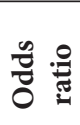 & & 1 & $\begin{array}{l}0 \\
\infty \\
0\end{array}$ & $\stackrel{m}{+}$ & $\stackrel{\Xi}{0}$ & $\stackrel{1}{0}$ & $\stackrel{+}{0}$ & & 1 & $\stackrel{\infty}{\stackrel{\infty}{-}}$ & ભે & $\stackrel{\infty}{\stackrel{\infty}{\sim}}$ & & 1 \\
\hline
\end{tabular}

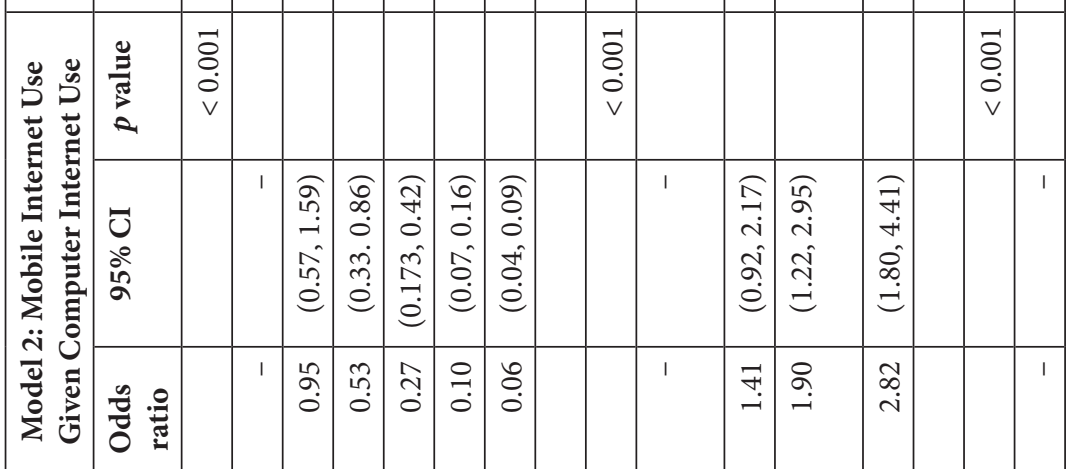

\begin{tabular}{|c|c|c|c|c|c|c|c|c|c|c|c|c|c|c|c|}
\hline$\stackrel{\mathscr{D}}{\mathscr{D}}$ & 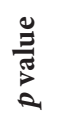 & $\begin{array}{l}\vec{\delta} \\
\dot{0} \\
\dot{0} \\
v\end{array}$ & & & & & & & $\begin{array}{l}\overrightarrow{8} \\
\dot{0} \\
\dot{0} \\
v\end{array}$ & & & & & $\begin{array}{l}\overrightarrow{8} \\
\dot{0} \\
\dot{0}\end{array}$ & \\
\hline 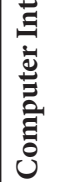 & $\begin{array}{l}0 \\
\text { 우 } \\
\text { in }\end{array}$ & & 1 & 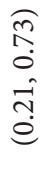 & $\begin{array}{c}0 \\
0 \\
0 \\
0 \\
0 \\
-1 \\
0 \\
0\end{array}$ & $\begin{array}{l}\hat{\sigma} \\
\tilde{0} \\
m \\
m \\
\stackrel{0}{0}\end{array}$ & $\begin{array}{c}\widehat{\vec{\jmath}} \\
0 \\
\hat{0} \\
\dot{0} \\
\dot{0}\end{array}$ & $\begin{array}{c}\overparen{\exists} \\
\overrightarrow{0} \\
\dot{0} \\
\dot{0} \\
\dot{0}\end{array}$ & & 1 & 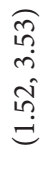 & 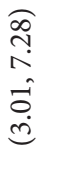 & 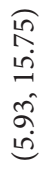 & & 1 \\
\hline 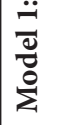 & 菅 & & 1 & ڤે & $\vec{n}$ & $\stackrel{\overbrace{}}{0}$ & $\stackrel{m}{\circ}$ & $\begin{array}{l}\hat{0} \\
\dot{0}\end{array}$ & & 1 & $\vec{m}$ & $\begin{array}{l}\infty \\
\dot{0} \\
\dot{+}\end{array}$ & $\begin{array}{l}\bullet \\
\stackrel{0}{0}\end{array}$ & & 1 \\
\hline 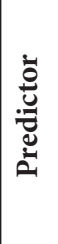 & & $\underset{z}{\infty}$ & $\begin{array}{l}\stackrel{H}{N} \\
\stackrel{\infty}{-1}\end{array}$ & $\begin{array}{l}m \\
\text { ñ } \\
\text { L }\end{array}$ & $\begin{array}{l}\vec{H} \\
1 \\
n \\
n\end{array}$ & $\begin{array}{l}5 \\
1 \\
10 \\
4\end{array}$ & $\begin{array}{l}+5 \\
1 \\
1 \\
n \\
n\end{array}$ & $\begin{array}{l}+ \\
0 \\
0\end{array}$ & 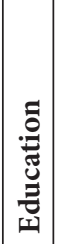 & 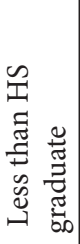 & 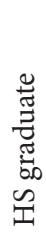 & 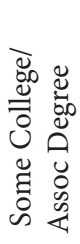 & 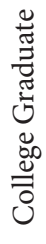 & 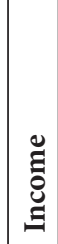 & 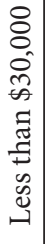 \\
\hline
\end{tabular}




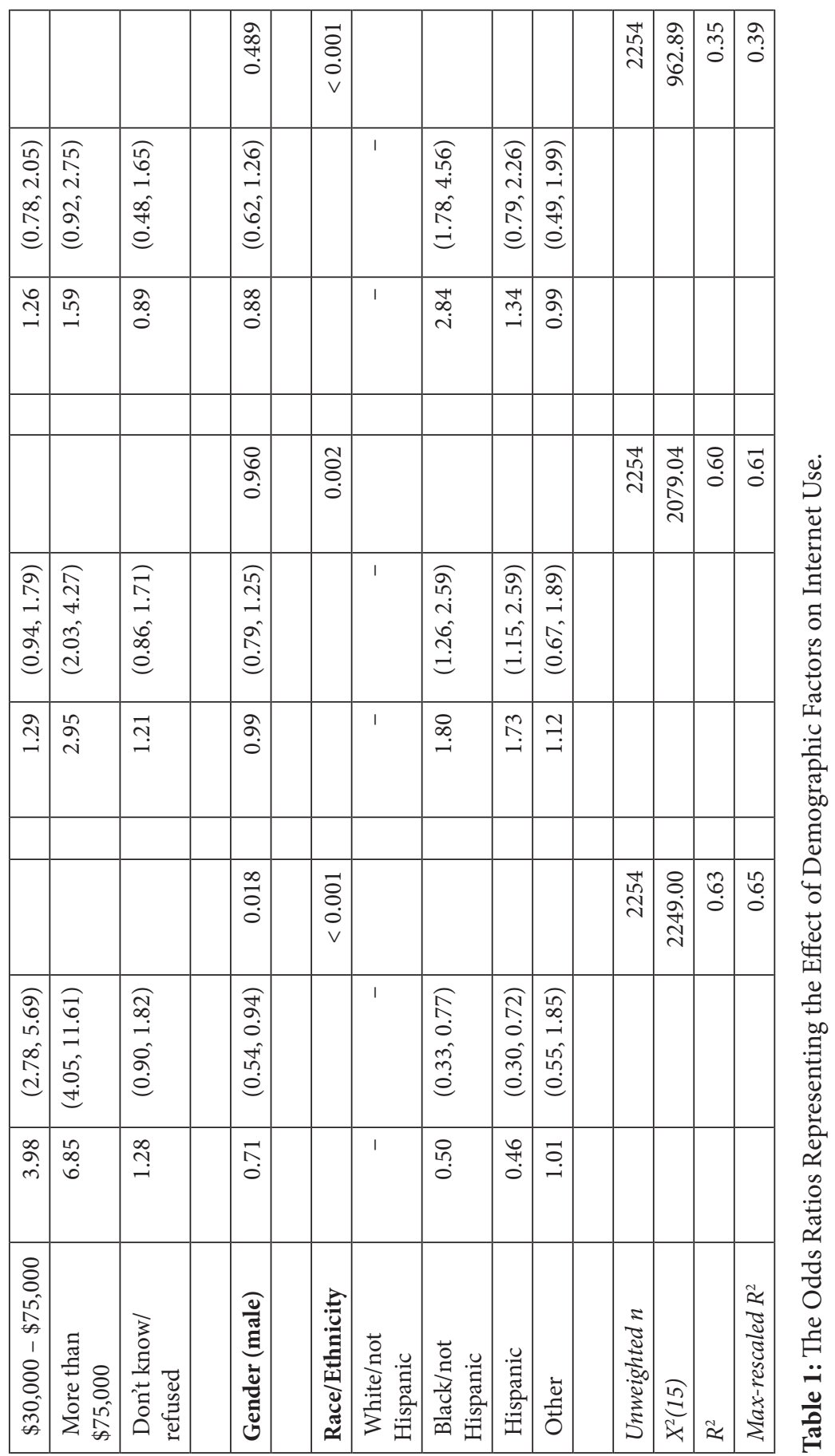


among those with less than a high school degree to $17.9 \%$ among those with some college/associates degree, but then declined among college graduates (9.4\%). Mobile-mostly Internet use among Black respondents was $23.5 \%$ compared to only $9.3 \%$ among White respondents.

The explanatory power of the demographic factors declines from an $R^{2}$ of 0.63 in Model 1, to 0.60 in Model 2, to finally 0.35 in Model 3. This suggests that demographics variables have more explanatory power when predicting computer Internet use and mobile Internet use than mobile-mostly Internet use, perhaps because other factors like familiarity with technology are more predictive of mobile-mostly Internet use.

\section{Do demographic characteristics have the same effect for each of these three transitions to a different level of Internet use?}

The expected age effect - Internet use declines with age - is the only effect that is consistent across all levels of Internet use. For example, compared to the youngest age group (18-24), being in the oldest age group (65 and older) reduces the odds of being a computer Internet user by $93 \%$; it similarly reduces the odds of being a mobile Internet user (conditional on computer Internet use) by $94 \%$; and it reduces the odds of being a mobile-mostly Internet user (conditional on mobile Internet use) by $96 \%$.

The expected education effect - Internet use increases with education - is not monotonic across all levels of Internet use. Compared to the lowest education group (less than high school degree), being in the highest education group (college degree) increases the odds of being a computer Internet user by a factor of 9.7; it increases the odds of being a mobile Internet user (conditional on computer Internet use) by a factor of 2.8; however, it has no significant effect on the odds of being a mobile-mostly Internet user (conditional on mobile Internet use). Similarly, the expected income effect - Internet use increases with income - is not monotonic across all levels of Internet use. Being in the highest income group has a positive effect on computer Internet use and mobile Internet use (Models 1 and 2), but it had no effect on becoming a mobile-mostly user (Model 3 ). This suggests that high-education and highincome adults make the transition to having Internet and make the transition to going online using their phones, but they stop short of relying on their phones to go online.

Gender was only associated with computer Internet use (Model 1), and not with the other two transitions. Race/ethnicity was the only characteristic that had the opposite effect on the first transition (computer Internet use) compared to the other two transitions (mobile Internet use and mobile-mostly Internet use). Non-Hispanic Black and Hispanic respondents are less likely to make the first transition to going online using a computer (Model 1), but conditional on Internet access they are more likely to make the second transition to using their 
phones to go online (Model 2), and conditional on mobile Internet use, Black respondents are more likely to make the final transition to use mostly a phone to go online (Model 3).

\section{Adding substantive variables}

The demographic differences between users and non-users reported above can lead to errors of nonobservation in surveys. However, these differences can be accounted for by standard weighting procedures. Therefore, the important issue is whether there are differences between users and non-users on nondemographic variables, and whether these differences persist after controlling for demographic differences.

Next, I fit a series of binary logit models, one for each level of Internet use, but this time I add to the baseline demographics model a series of three nondemographic variables (indicators of quality of life, social trust, and tablet ownership). Table 2 shows the odds ratios for each variable. As before, I conditioned on the appropriate subsamples of the data for each model. Since the baseline models from Table $\mathbf{1}$ are nested in these new models, I am able to conduct likelihood ratio tests. The log likelihood ratio test-statistic, which is Chi-square distributed (with three degrees of freedom), is shown in the last row of Table 2.

For Model 1, the likelihood ratio test reveals that the addition of the nondemographic variables adds significantly to the model, over and above the demographic controls $\left(\mathrm{X}^{2}(3)=200.9, p<0.001\right)$. Tablet use was significantly associated with Internet use; computer Internet users are 5.6 times more likely to use a tablet compared to computer Internet non-users.

For Model 2, the addition of the non-demographic variables also adds significantly to the model $\left(\mathrm{X}^{2}(3)=587.7, p<0.001\right)$. As with Model 1 , this new model reveals that of the three predictors only tablet use is significantly associated with mobile Internet use, but it produces a relatively strong effect: mobile Internet users are 9.2 times more likely to use a tablet compared to non-users of mobile Internet.

For Model 3, once again the non-demographic variables add significantly to the model $\left(\mathrm{X}^{2}(3)=53.8, p<0.001\right)$. And once again tablet use was the lone significant predictor; mobile-mostly Internet users are 1.8 times more likely to use a tablet compared to computer-mostly Internet users.

I used a rather limited set of five demographic controls. But when I added to the model a larger set of demographic variables (marital status, employment status, regions, and urbanicity), tablet use was still significantly associated with each of the levels of Internet use. These results suggest that coverage error may affect the accuracy of at least some estimates based on online surveys designed for computers. For example, if traditional Web surveys disproportionately omit mobile-mostly users (by blocking them or using a non-optimized survey), then 


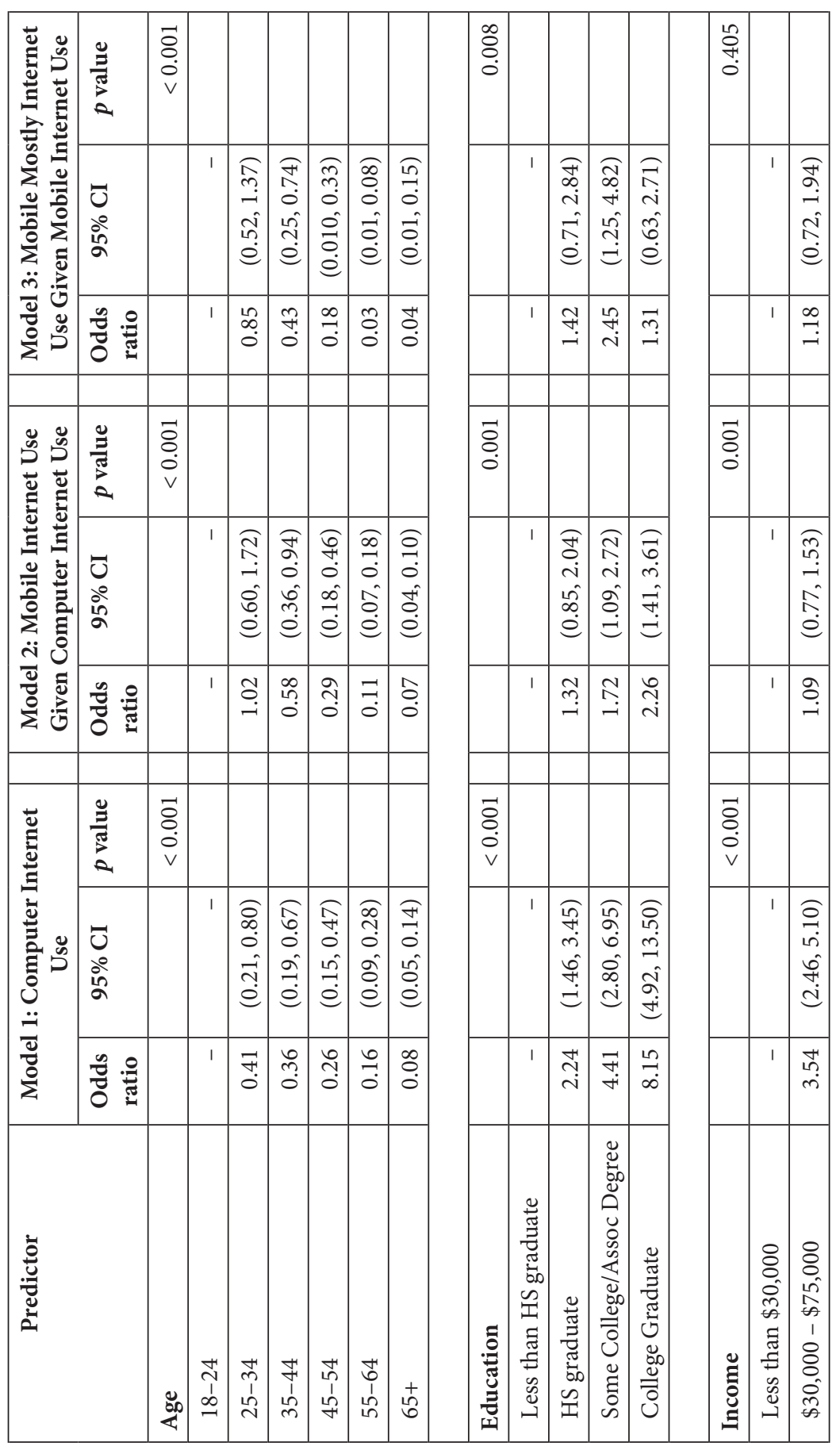




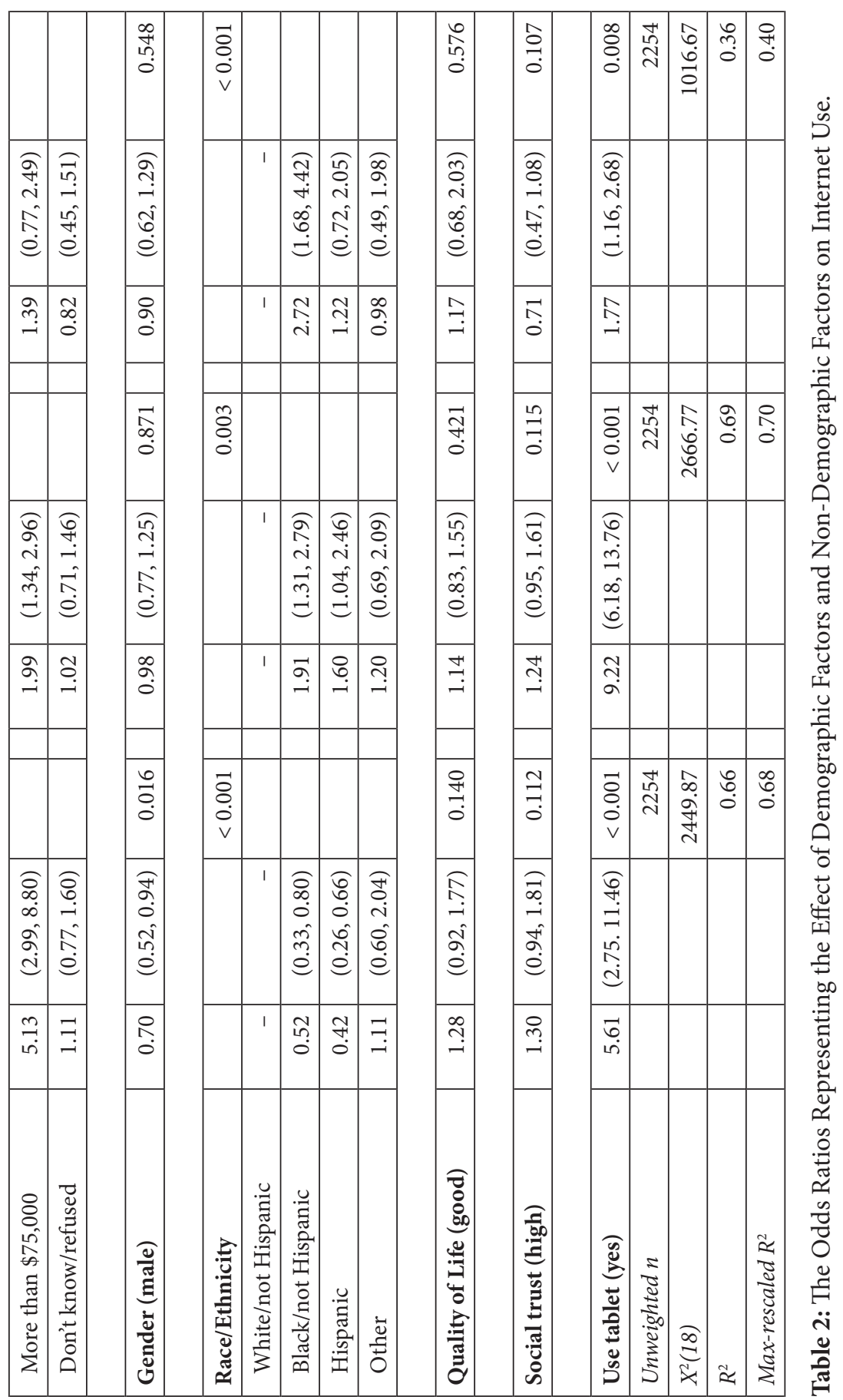


their absence from the sample has the potential to affect some estimates based on Web surveys (e.g., estimates of tablet use).

\section{Discussion}

This chapter offered a new way to conceptualize Internet use as a series of three transitions, each involving a jump from one side to the other side of a digital divide. It reported on the effect of demographic characteristics on each step in this process using a sample of US respondents. There are three main conclusions. First, at each stage there are significant differences between those on either sides of the divide. Starting with the digital divide, computer Internet use is uneven across various demographic groups, with younger, highly educated, affluent, and White adults more likely to use the Internet than others. The device divide was also apparent: mobile Internet use was unevenly distributed across demographics groups. In addition, the usage divide is reflected in the significant demographic differences between those who use mostly their phones to go online and those who use mostly their computers.

Second, I found that some demographic characteristics had different effects on the three transitions, which highlights the fact that each type of digital divide is unique. One rule of thumb for Internet use - that it decreases with age still holds when considering all three levels of Internet use. Younger adults are more likely to make all three transitions; that is, from a non-user to a computer Internet user, then to a mobile Internet user, and then finally to becoming a mobile-mostly user. However, other rules of thumb - that Internet use increases with education or income - appear to be true for the first two transitions, but not when considering mobile-mostly use. In addition, the notion that non-Hispanic Black and Hispanic adults are less likely to use the Internet compared to White adults was only true for the first transition. It shifted when considering mobile Internet use and mobile-mostly Internet use; conditional on computer Internet use, non-Hispanic Black and Hispanic adults are actually more likely to make the transition to going online using their phones and Black adults were reliably more likely to become mobile-mostly users compared to White adults. Generally speaking, in the US the device divide looks a lot like the digital divide in terms of the demographics characteristics of people on either side, but the usage divide is quite unique. This is perhaps not surprising given the nature of the divides. While the first two divides have to do with adoption of a technology (computers and Internet-enabled phones), the third divide has to do with preferences toward a device that one is already using. Given the recent trends, the first two transitions seem almost inevitable for those who rely on technology while the third transition may not become widespread, given that most people prefer to use computers rather than their phones for complicated and long tasks (Robinson 2014). Of course this could change 
as phones evolve to feature larger screens (e.g., phablets) and more advanced computing capabilities.

Third, I found that one non-demographic variable - tablet use - was significantly associated with each of the transitions over and above demographic variables. This suggests that survey practitioners cannot correct all of the bias from omitting certain groups of Internet users by weighting on demographics characteristics. By contrast, the other variables - life quality and social trust - were not associated with the transitions over and above the demographics controls, suggesting that differences between those who are covered and not covered on these two particular survey variables can be accounted for by weighting on demographics characteristics. Perhaps variables related to technology are the ones that are more likely to be biased because they are likely to be correlated with Internet use. Future work should explore this for a larger set of survey variables of interest. In the meantime, to reduce potential coverage errors, online survey researchers would be wise to try to accommodate all online users if possible through use of a hybrid or adaptive design approach that optimizes their questionnaires for small devices.

It should be noted that this study used data from a telephone survey with a relatively low response rate, which may increase the risk of nonresponse bias. Nonetheless, this analysis did use weighting adjustments to help account for any potential nonresponse errors. That said, it would certainly be beneficial to conduct more research on this topic using a different survey mode (e.g., faceto-face) that could achieve higher response rates. That would also eliminate the possibility for differential measurement errors in the answers provided by landline and cell respondents. It should also be pointed out that the analysis uses cross-sectional data to observe aggregate level but not individual level change, the latter of which would be possible to observe if future researchers were to grapple with this same research topic using longitudinal data.

Moving forward, it is clear that the discussion about coverage bias in Web surveys will need to focus not only on the digital divide but also on the device divide and on the emerging divide between the growing number of individuals who prefer using their phone to go online and those who prefer to use their computer. This shift in Internet use is affecting errors of non-observation in Web surveys in new and complicated ways. The effects will continue to be influenced by the proportions of people in each Internet use group, the size of differences between users and non-users, and whether these differences can be accounted for by demographic variables.

\section{Acknowledgements}

I acknowledge the Pew Reseach Center for providing access to the data used in this analysis. They bear no responsibility for the interpretations or conclusions reached based on my analysis. 


\section{References}

AAPOR. (2014). Mobile Technologies for Conducting, Augmenting and Potentially Replacing Surveys: Report of the AAPOR Task Force on Emerging Technologies in Public Opinion Research. Deerfield, IL: American Association for Public Opinion Research.

Antoun, C., \& Couper, M. P. (2013, November). Mobile-Mostly Internet Users and Selection Bias in Traditional Web Surveys. Paper presented at the annual meeting of the Midwest Association for Public Opinion Research, Chicago, USA.

Bethlehem, J. (2010). Selection Bias in Web Surveys: Selection Bias in Web Surveys. International Statistical Review, 78(2), 161-188. DOI: http:// dx.doi.org/10.1111/j.1751-5823.2010.00112.x

Blumberg, S. J., \& Luke, J. V. (2013). Wireless Substitution: Early Release of Estimates From the National Health Interview Survey, January-June 2013. Center for Disease Control Report. Retrieved from http://www.cdc.gov/ nchs/data/nhis/earlyrelease/wireless201312.pdf

Couper, M. P., Kapteyn, A., Schonlau, M., \& Winter, J. (2007). Noncoverage and Nonresponse in an Internet Survey. Social Science Research, 36(1), 131-148. DOI: http://dx.doi.org/10.1016/j.ssresearch.2005.10.002

Curtin, R., Presser, S., \& Singer, E. (2005). Changes in telephone survey nonresponse over the past quarter century. Public opinion quarterly, 69(1), 87-98.

de Bruijne, M., \& Wijnant, A. (2014). Mobile Response in Web Panels. Social Science Computer Review, 32(6), 728-742. DOI: http://dx.doi. org/10.1177/0894439314525918

Dever, J. A., Rafferty, A., \& Valliant, R. (2008). Internet Surveys: Can Statistical Adjustments Eliminate Coverage Bias. Survey Research Methods, 2, $47-62$.

Duggan, M., \& Rainie, L. (2012). Cell Phone Activities 2012. Pew Research Center's Internet \& American Life Project.

Duggan, M., \& Smith, A. (2013). Cell Internet Use 2013. Pew Research Center's Internet \& American Life Project.

Fuchs, M., \& Busse, B. (2009). The Coverage Bias of Mobile Web Surveys across European Countries. International Journal of Internet Science, 4(1), 21-33.

Groves, R. M., \& Peytcheva, E. (2008). The impact of nonresponse rates on nonresponse bias a meta-analysis. Public opinion quarterly, 72(2), 167-189. DOI: http://dx.doi.org/10.1093/poq/nfn011

Lenhart, A. (2003). The Ever-Shifting Internet Population: A New Look at Internet Access and the Digital Divide. Washington, DC: The Pew Internet and American Life Project.

Lipsman, A., \& Aquino, C. (2013). Mobile Future in Focus. comScore Report. Retrieved from http://www.comscore.com/Insights/Presentations-andWhitepapers/2013/2013-Mobile-Future-in-Focus 
Metzler, A., \& Fuchs, M. (2014, December). Coverage Error in Mobile Web Surveys Across European Countries. Paper presented at the Internet Survey Methodology Workshop, Bozen-Bolzano, Italy.

Mohorko, A., de Leeuw, E., \& Hox, J. (2013). Internet Coverage and Coverage Bias in Europe: Developments Across Countries and Over Time. Journal of Official Statistics, 29(4), 609-622. DOI: http://dx.doi.org/10.2478/jos-20130042

NTIA (National Telecommunications and Information Administration). (1995, July). Falling Through the Net: A Survey of the »Have Nots" in Rural and Urban America.

Pearce, K. E., \& Rice, R. E. (2013). Digital Divides From Access to Activities: Comparing Mobile and Personal Computer Internet Users. Journal of Communication, 63(4), 721-744. DOI: http://dx.doi.org/10.1111/jcom.12045

Peterson, G. (2012, May). Unintended Mobile Respondents. Paper presented at the CASRO Technology Conference, New York, USA.

Pew Center for the People and the Press. (1995). Technology in the American household; Americans going online... Explosive growth, uncertain destinations. Retrieved from http://www.people-press.org/1995/10/16/americansgoing-online-explosive-growth-uncertain-destinations/

Pew Research Center. (2012). Spring 2012 tracking survey [Questionnaire and Topline report]. Retrieved from http://www.pewinternet.org/files/oldmedia/Files/Questionnaire/2012/PIP_Just_In_Time_topline.pdf

Poushter, J., Bell, J., \& Oates, R. (2015). Internet Seen as Positive Influence on Education but Negative on Morality in Emerging and Developing Nations. Pew Research Center.

Revilla, M., Toninelli, D., Ochoa, C., \& Loewe, G. (2014). Do Online Access Panels Really Need to Allow and Adapt Surveys to Mobile Devices? Barcelona: Universitat Pompeu Fabra, RECSM Working Paper Number 41. Retrieved from http://www.upf.edu/survey/_pdf/RECSM_wp041.pdf

Robinson, O. (2014). Finding Simplicity in a Multi-Device World. GfK online article. Retrieved from http://blog.gfk.com/2014/03/finding-simplicity-ina-multi-device-world

Smith, A. (2012). Nearly Half of American Adults Are Smartphone Owners. Pew Research Center.

Tourangeau, R., Conrad, F., \& Couper, M. (2013). The Science of Web Surveys. Oxford: Oxford University Press. DOI: http://dx.doi.org/10.1093/acpr of:oso/9780199747047.001.0001

Townsend, L., \& Rios, H. (2011)..Defragmenting Mobile Research - How to Successfully Combine the Wide Array of Available Mobile Tools and Create a Highly Effective Mobile Platform. Kinesis Survey Software White Paper. Retrieved from http://www.kinesissurvey.com/files/DefragmentingMobileResearch_KinesisWhitepaper.pdf

Zickuhr, K. (2013). Who's not online and why. Pew Research Center. 